\title{
Spraying Beta: Information Behavior in the Small World of Rock Climbing
}

\author{
Bree Norlander \\ University of Washington \\ Information School \\ norlab@uw.edu
}

\begin{abstract}
Introduction
Observations at an indoor rock climbing gym reveal Elfreda Chatman's use of the small world concept in action. The behaviors of the "legitimized others," (Chatman, 1999) or what I will call the core community of climbers can be analyzed through the four concepts of Chatman's Theory of Normative Behavior: Social Norms, Worldview, Social Types, and Information Behavior (Burnett, Besant, \& Chatman, 2001). I will discuss each concept in turn, and model a climber's information behavior as it pertains to climbing a selected route. I will also be drawing on sociological research into subculture, which shares many of the characteristics of Chatman's small world.
\end{abstract}

\section{Social Norms}

Each climbing location may have its own set of social norms, but a Google search for "climbing etiquette" reveals similarities across locations. The climbers in the core community at the gym I frequent adhere to an unspoken dress code, albeit with broad parameters, and an unspoken code of behavior. The members of this core community wear comfortable, relatively modest clothing, including shirts rather than bare chests (men) or midriffs (women). However, the shirt or no shirt etiquette is a topic of debate in the climbing world (see Hedgingwendy, n.d.) and certain climbers get away with flaunting these norms if they are well regarded within the core community. Participants of the core climber community own the "right" climbing shoes. Michelle Donnelly explains:

Real or authentic subcultural identities are closely tied to knowing what products to buy or not buy, whereas, at the same time, the consumption of products-the wrong products or consumption in the wrong ways-is often an identifying feature of more peripheral participants or of nonparticipants. (2006, p. 219-220)

Friendliness and politeness are valued in the core community. However, providing "beta" (information on how to climb a specific route) unsolicited is a serious faux pas (Wingo, 2012). Climbers who make this a habit are known as "beta sprayers" and risk exclusion from the core community. Additionally, the core community respects the presence of children and frowns upon swearing and yelling.

Despite the few strict norms, the climbing culture at this gym is supportive and climbers regularly cheer on fellow climbers even if they are not acquainted. Sharing of beta is appreciated when solicited and adds to the air of camaraderie. 


\section{Worldview}

Despite its evolving culture, Don Mellor explains, "American climbing has always been an endeavor infused with meaning. It has been a culture bonded by some common understandings. Its symbols. . . are ritual emblems of entry into its proudly select community" (2001, p. 39).

One of the climbing community's common understandings is its unique vocabulary, which is drawn upon heavily when exchanging information about how to climb a route. Knowledge of the vernacular identifies a climber as a potential "insider" of the core community or subculture (Haenfler, 2013, p. 18).

\section{Social Types}

Climbers are generally "typed" based on the difficulty of the routes they can climb. Bouldering (climbing shorter routes without ropes) routes in this gym range in difficulty level from VB/V0 (easiest) - V10 (most difficult). A climber may refer to herself or another as a "V5" climber for example. This is an indication of both the climber's ability and commitment to the sport, with higher numbers being more desirable. Those who climb harder routes have more flexibility when it comes to social norms and are trusted more for their beta, consistent with Chatman's theory that the information from a person of desired type is more readily accepted and disseminated (2001).

\section{Information Behavior}

The behavior model below addresses the information behavior of a climber approaching the decision to climb a selected route. When climbers approach a climbing route, they have four options: climb, intentionally avoid information from others, receive unsolicited information, or seek information.

Information avoidance has often been conceptualized as pathological in the information seeking literature (Manheim, 2014). Much of the research focuses on health information avoidance, which is often motivated by a fear of experiencing negative emotions (Sairanen \& Savolainen, 2010). But the climber's motivation to avoid information is far from fear induced. Moja explains, "many individuals approach climbing as a puzzle and may prefer to fit the pieces together on their own" (2014, para. 11).

Beta spraying can frustrate a climber who desires to "solve" the climb without external information. There is little information on this type of information behavior, but it bears resemblance to Fisher's idea of the "hostage phenomenon," when one is forced to hear information due to proximity (Fisher \& Naumer, 2006).

Information seeking in this context can consist of one or more of the following: using information available on the route (visually assessing the holds and noting the location of chalk from previous climbers), asking another climber for information, 
consulting a written description of the route and the grade, or observing another climber's movements as he attempts the route.

Next, the climber will either attempt to climb, or choose not to climb. If she attempts to climb the route and falls off or gets to a point in which she can't continue, she can cycle back through the information behavior used before the attempt, use trial and error, or discontinue her attempts. Ultimately, she will succeed in completing the route or end her attempts.

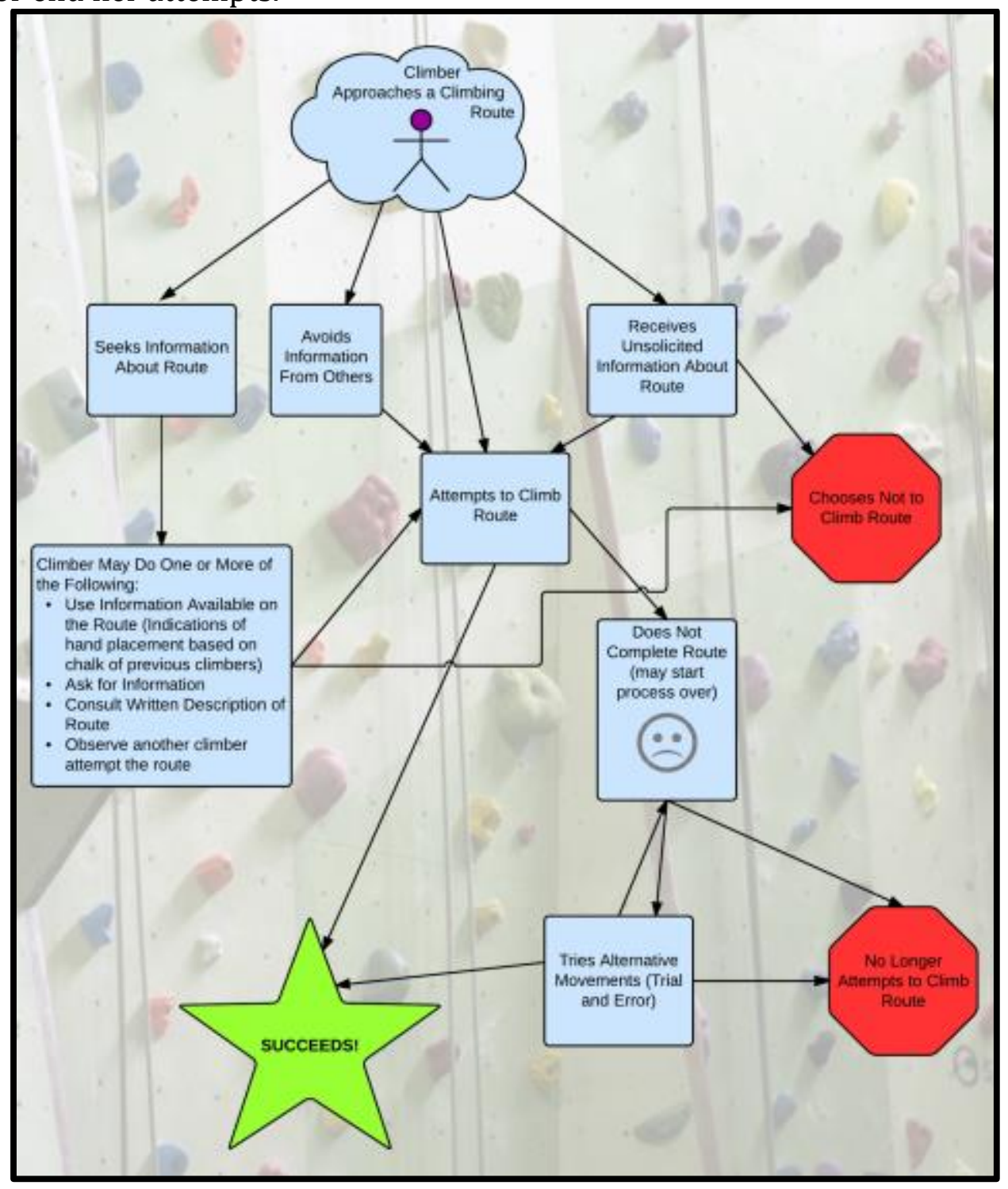

\section{Further Study}

Currently there is little research into the benefit of information avoidance in learning a skill. Manheim writes, "avoidance literature has primarily focused on information that is perceived to be undesirable-or at least has the potential to be so" $(2014$, p. 6). Research should be undertaken to study the avoidance of desirable information and the motivation for such avoidance. There is also room for research 
into forced information and the negative interpersonal impact of unsolicited information, as well as how this could hinder the learning process.

\section{Conclusion}

The small world of climbers may have unique vernacular and specialized social norms, but it is not unique in its information behavior. This model can also be applied to a crafter attempting a new project, a student completing a math problem, or a cook trying a new recipe. This information behavior model is a starting point for understanding decision-making as it pertains to learning in a broader context. 
References

Burnett, G., Besant, M., \& Chatman, E. A. (2001). Small worlds: Normative behavior in virtual communities and feminist bookselling. Journal of the American Society for Information Science \& Technology, 52(7), 536-547.

Chatman, E. A. (1999). A theory of life in the round. Journal Of The American Society For Information Science, 50(3), 207-217.

Donnelly, M. (2006). Studying extreme sports beyond the core participants. Journal of Sport and Social Issue, 30 (2), 219-224.

Fisher, K. E., \& Naumer, C. M. (2006). Information grounds: Theoretical basis and empirical findings on information flow in social settings. In A. Spink \& C. Cole (Eds.), New directions in human information behavior (pp. 93-111). Amsterdam: Kluwer

Haenfler, R. (2013). Subcultures the basics. Hoboken: Taylor and Francis. Retrieved from http://www.123library.org/book details/?id=110432

Hedgingwendy. (n.y., August 1) Shirts vs. skins: What's your take on rock climbing gym etiquette? [Web log post]. Retrieved from http://heroes.climbfind.com/post/28485571125/shirts-vs-skins-whatsyour-take-on-rock

Manheim, L. (2014). Information non-seeking behaviour. Information Research, 19 (4), 210-220.

Mellor, D. (2001). American rock: Region, rock, and culture in American climbing. Woodstock, Vt: Countryman Press.

Moja. (2014, May 8). Seven mistakes to avoid as a new climber [Web log post]. Retrieved from http://mojagear.com/learn/2014/05/08/seven-mistakes-toavoid-as-a-new-climber/

Sairanen, A. \& Reijo S. (2010). Avoiding health information in the context of uncertainty management. Information Research, 15 (4): 7.

Wingo, Aubrey. (2012, April 4). Ethics - The dos and don'ts of rock climbing [Web log post]. Retrieved from http://aiguilleclimbing.blogspot.com/2012/04/ethics-dos-and-donts-ofrock-climbing.html 